\title{
Approximate Query Answering Using Data Warehouse Striping
}

\author{
JORGE R. BERNARDINO \\ Polytechnic of Coimbra, ISEC, DEIS, Apt. 10057, P-3030-601 Coimbra, Portugal \\ jorge@isec.pt \\ PEDRO S. FURTADO \\ HENRIQUE C. MADEIRA \\ pnf@dei.uc.pt \\ henrique@dei.uc.pt
}

University of Coimbra, DEI, Pólo II, P-3030-290 Coimbra, Portugal

\begin{abstract}
This paper presents and evaluates a simple but very effective method to implement large data warehouses on an arbitrary number of computers, achieving very high query execution performance and scalability. The data is distributed and processed in a potentially large number of autonomous computers using our technique called data warehouse striping (DWS). The major problem of DWS technique is that it would require a very expensive cluster of computers with fault tolerant capabilities to prevent a fault in a single computer to stop the whole system. In this paper, we propose a radically different approach to deal with the problem of the unavailability of one or more computers in the cluster, allowing the use of DWS with a very large number of inexpensive computers. The proposed approach is based on approximate query answering techniques that make it possible to deliver an approximate answer to the user even when one or more computers in the cluster are not available. The evaluation presented in the paper shows both analytically and experimentally that the approximate results obtained this way have a very small error that can be negligible in most of the cases.
\end{abstract}

Keywords: data warehousing, distributed query optimization, data partitioning, performance optimization, approximate query answering

\section{Introduction}

Data warehousing refers to "a collection of decision support technologies aimed at enabling the knowledge worker (executive, manager, analyst) to make better and faster decisions" (Chauduri and Dayal, 1997). A data warehouse is a global repository that stores large amounts of data that has been extracted and integrated from heterogeneous, operational or legacy systems. OLAP (on-line analytical processing) is the technique of performing complex analysis over the information stored in a data warehouse (Codd et al., 1993). The data warehouse coupled with OLAP enables business decision makers to creatively analyze and understand business trends since it transforms operational data into strategic decision making information. Typical warehouse queries are very complex and ad hoc and generally access huge volumes of data and perform many joins and aggregations. Efficient query processing is a critical requirement for data warehousing because decision support applications typically require interactive response times.

In this paper, we assume that a multidimensional database is based on a relational data warehouse in which the information is organized in a star schema (Kimball, 1996), as illustrated in figure 1 . 


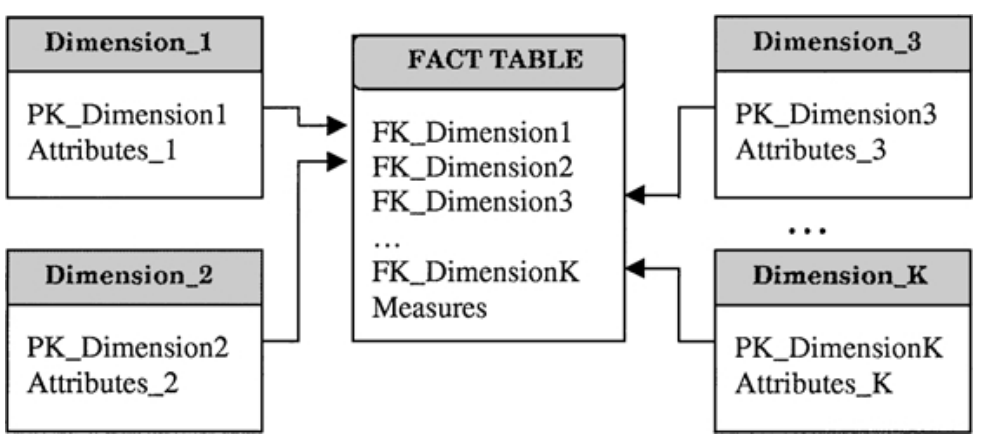

Figure 1. Star schema.

A star schema is composed by a set of dimension and fact tables where the fact table accounts for most of the space occupied by all the tables in the star for most of the cases (Kimball et al., 1998). However, this table is highly normalized, which means that it really represents the most effective (concerning storage space) relational way to store the facts. The dimension tables are very denormalized, but these tables usually represent a small percentage of the space in the star. The star schema is also particularly optimized for the execution of complex queries that aggregate a large amount of data from the fact table.

The data striping technique uses the well-know round-robin partitioning approach applied to the very low level of the data warehouse facts in such a way that assures uniform distribution of the partitioned data. This very simple and old partitioning technique proved to be very effective for the typical queries in data warehouses. In fact, using this partitioning technique we can convert one query into queries that compute partial results, and the global result can be computed very fast from these partial results. The (partial) queries are executed independently in the different computers, which is the necessary condition to achieve optimal load balance and performance scaleup. This data partitioning technique, called "Data Warehouse Striping" (DWS), was proposed in a previous work (Bernardino and Madeira, 2000).

In spite of the potential dramatic speedup and scaleup that can be achieved by using the DWS technique, the fact that the data warehouse is distributed over a large number of workstations (nodes) greatly limits the practical use of the technique. The probability of having one of the workstations in the system momentarily unavailable cannot be neglected for a large number of nodes. The obvious solution of building the system using fault tolerant computers is very expensive and will contradict the initial assumption of DWS technique of using inexpensive workstations with the best cost/performance ratio. Nevertheless, high availability is required for most data warehouses, especially in areas such as e-commerce, banks, and airlines where the data warehouse is crucial to the success of the organizations.

In this paper, we propose a new approximate query answering strategy to handle the problem of temporarily unavailability of one or more computers in a large data warehouse implemented over a large number of workstations (nodes) using the DWS technique. In the proposed approach the system continues working even when a given number of nodes are unavailable. The partial results from the available nodes are used to return an estimation 
of the results from the unavailable nodes. Currently, we provide approximate answers for typical aggregation queries providing the user with a confidence interval about the accuracy of the estimated result. The analytic and experimental study presented in this paper show that the error introduced in the query results can be really very small.

The rest of the paper is organized as follows. In the next section, we give an overview of related work and discuss the problems associated with approximate answering in data warehouses. Section 3 describes the DWS approach and Section 4 discusses approximate query answering using DWS in the presence of node failures. Section 5 analyzes the experimental results and the final section summarizes the conclusions from this work.

\section{Related work}

Statistical techniques have been applied to databases in different tasks for more than two decades (e.g. selectivity estimation (Selinger et al., 1979)). Traditionally, researchers are interested in obtaining exact answers to queries, minimizing query response time and maximizing throughput. In this work, we are interested in analyzing and giving solutions to the failure of one or more workstations in a DWS system. Thus, it has some similarities with approximate query answering research, where the main focus is to provide fast approximate answers to complex queries that can take minutes, or even hours to execute. In approximate query answering the size of the base data is minimized using samples, which is analogous to the failure of a workstation inhibiting the access to the part of the base data that resides in that workstation.

Recently, there has been a significant amount of work on approximate query answering (Acharaya et al., 2000; Haas, 1997; Vitter and Wang, 1999). One of the first works was (Hellerstein et al., 1997) where the authors proposed a framework for approximate answers of aggregation queries called online aggregation, in which the base data is scanned in random order at query time and the approximate answer is continuously updated as the scan proceeds. The Approximate Query Answering (AQUA) system (Gibbons and Matias, 1998) provides approximate answers using small, pre-computed synopses of the underlying base data. Other systems support limited on-line aggregation features; e.g., the Red Brick system supports running COUNT, $A V G$, and SUM (Hellerstein et al., 1997).

There has also been a considerable amount of work in developing statistical techniques for selectivity estimation and, more recently, for data reduction in large data warehouses. The major classes of techniques used are sampling (Hou and Taneja, 1988; Lipton et al., 1990; Haas et al., 1994; Lipton and Naughton, 1995; Haas et al., 1995; Ganguly et al., 1996), histograms (Kooi, 1980; Poosala et al., 1996; Poosala, 1997; Poosala et al., 1999), parametric modeling (Chen and Roussopoulos, 1994) and wavelets (Vitter and Wang, 1999). In Barbara et al. (1997) we have a survey of data reduction techniques that can be used for a variety of purposes including approximate query answering. In Gibbons and Matias (1998a) it is shown a framework for studying synopsis data structures for massive data sets and introduced two sampling-based synopses, concise samples and counting samples, that can be used to obtain larger samples for the same space and to improve approximate query answers for hot list queries. Maintenance algorithms exist for samples (Olken and Rotem, 1992; Gibbons et al., 1997a; Gibbons and Matias, 1998a) and histograms 
(Gibbons et al., 1997b). However, these maintenance techniques are only applicable to "base" statistics.

A large body of work exists in applying parallel processing techniques to relational database systems (Lu et al., 1994; DeWitt and Gray, 1992). The basic idea behind parallel databases is to carry out evaluation steps in parallel whenever possible, in order to improve performance. Two types of parallelism can be explored at the query level: inter-query parallelism (DeWitt et al., 1990), wherein multiple transactions are executed in parallel in a multiprocessor environment, and intra-query parallelism (Stonebraker et al., 1998), where several processors cooperate to concurrently execute a single SQL statement. The parallelism is used to improve performance through parallel implementation of various operations such as loading data, building indexes and evaluating queries. One of the first works to propose a parallel physical design for the data warehouse was Datta et al. (1998). In this work the author suggests a vertical partitioning of the star schema including algorithms but without quantifying potential gains.

Our work is also related to distributed processing in data warehouses. The fact that many data warehouses tend to be extremely large in size (Chauduri and Dayal, 1997) and grow quickly means that a scalable architecture is crucial. In spite of the potential advantages of distributed data warehouses, especially when the organization has a clear distributed nature, these systems are always very complex and have a difficult global management (Albrecht et al., 1998). On the other hand, the performance of distributed queries is normally poor, mainly due to load balance problems.

In this context, the DWS concept provides a flexible approach to distribution, inspired in both distributed data warehouse architecture and classical round-robin partitioning techniques. The data is partitioned in such a way that the load is uniformly distributed to all the available workstations and, at the same time, the communication requirements between workstations is kept to a minimum during the query computation phase. This paper marries the concepts of distributed processing and approximate query answering to provide a fast and reliable relational data warehouse.

\section{Data warehouse striping}

Star schemas provide intuitive ways to represent the typical multidimensional data of businesses in a relational system. In the data warehouse striping (DWS) approach, the data of each star schema is distributed over an arbitrary number of workstations having the same star schema (which is the schema of the equivalent centralized version). The dimension tables are replicated in each machine (i.e., each dimension has exactly the same rows in all the workstations) and the fact data is distributed over the fact tables of each workstation using a strict row-by-row round-robin partitioning approach (see figure 2).

This data partitioning for star schemas balances the workload by all computers supporting parallel query processing as well as load balancing for disks and processors. The replication of dimension tables doesn't represent a serious overhead because usually the dimensions only represent less than $10 \%$ of the space occupied by all database (Kimball, 1996). With this approach typical OLAP queries are executed in parallel by all the computers of the DWS system. 


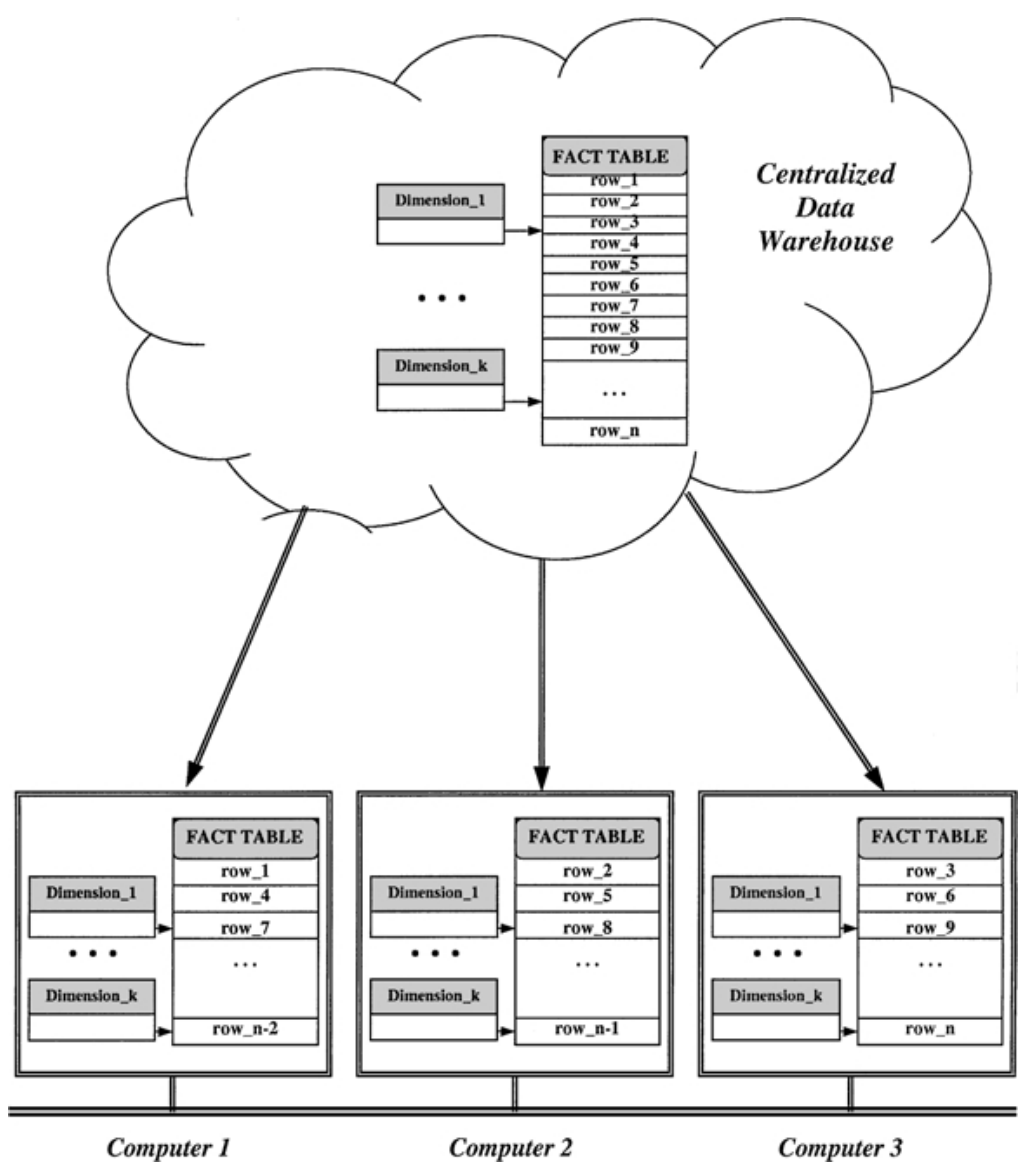

Figure 2. Data warehouse striping.

An important aspect of the DWS technique is that it ensures a uniform distribution of fact data over all computers as this distribution is in fact a random sample method called systematic sampling (Cochran, 1977) as we will see in the next section.

\subsection{Uniform random samples}

In DWS context the possibility to obtain random samples is essential because the system returns approximate query answers when some of the computers are not available and in that case we can compute the error in the estimates in the form of confidence intervals.

The systematic sampling method has as sample basis one file or the list of elements of the population, satisfying the hypothesis $M=N \cdot n$, where $M$ is the population size and $N$ is a number that belongs to the natural number set (in our case represents the number of computers used by DWS system) and $n$ is the sample size. 
The procedure to apply the systematic sample method consists in choosing randomly one number $k$ in the interval $[1, N]$, which serves as seed and the first element of the sample. The systematic sample is composed by the elements with the following numbers $k, k+N, k+2 N, \ldots, k+(n-1) N$. If we have a population $X_{1}, X_{2}, \ldots, X_{M}$ and if we want to choose a systematic samples of this population $(M=N \cdot n)$, we can write

$$
\begin{aligned}
& \begin{array}{lllll}
X_{1} & X_{1+N} & X_{1+2 N} & \cdots & X_{1+(n-1) N}
\end{array} \\
& X_{2} \quad X_{2+N} \quad X_{2+2 N} \cdots X_{2+(n-1) N}
\end{aligned}
$$

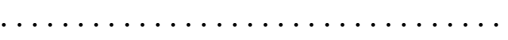

$$
\begin{aligned}
& X_{N} \quad X_{N+N} \quad X_{N+2 N} \cdots X_{N+(n-1) N}
\end{aligned}
$$

Each line is a systematic sample draw with $n$ elements, selected from every $N$ th element. In our case, each of these lines represents the fact rows in each one of the $N$ computers of the DWS system. This means the DWS technique uses a probabilistic sample method that is not biased due to its theoretical characteristics based on probabilistic theory (Hansen et al., 1953). Another advantage is the possibility to compute the degree of uncertainty, i.e. the error of the estimate, in the form of confidence intervals. This is only due to the fact we have uniform random samples.

To demonstrate empirically this characteristic we will use real data to show the roundrobin data partitioning technique of DWS is uniform, i.e., the data is uniformly distributed over the computers. Consequently, if one or more computers of DWS system are not available, this is not a critical situation because the values in the others have approximately the same statistical behavior.

In these experiments we use the close value of Dow-Jones stock index for the last 40 years. We collect the daily close value of Dow-Jones from April 13th 1961 until December 20th 2000 , which corresponds to 10,000 values. Each value represents one fact of a hypothetical star schema representing Dow Jones stock index. The temporal data distribution is represented in figure 3 , which corresponds to store stock index values in only one computer, denoted PC_0.

Supposing that we are using 10 computers, using the DWS technique to partition the Dow-Jones index, the figure 4 shows the values stored in each one of the computers (PC_1 to $P C_{-}$10). In this case each computer have 1000 values of Dow-Jones stock index.

To go through our point of view, we had divided the stock index values over 100 computers and the figure 5 presents the graphics of the values in each computer (PC_1 to PC_100). In this case each computer only have 100 values of Dow-Jones stock index.

As we can see, the curves of the values in each computer are very similar, which shows the partitioning technique used by DWS leads to an uniform data distribution over the computers. Additionally, comparing the figures 4 and 5 with figure 3 , we see they are very similar to each other meaning the samples contained in each computer have the same distribution of the population from where they derive.

The result of these experiments reinforces our confidence that DWS technique follow a uniform distribution, which have positive effects in the precision of the estimates, when we have computers that are not available. This approach guarantees that the set of tuples 


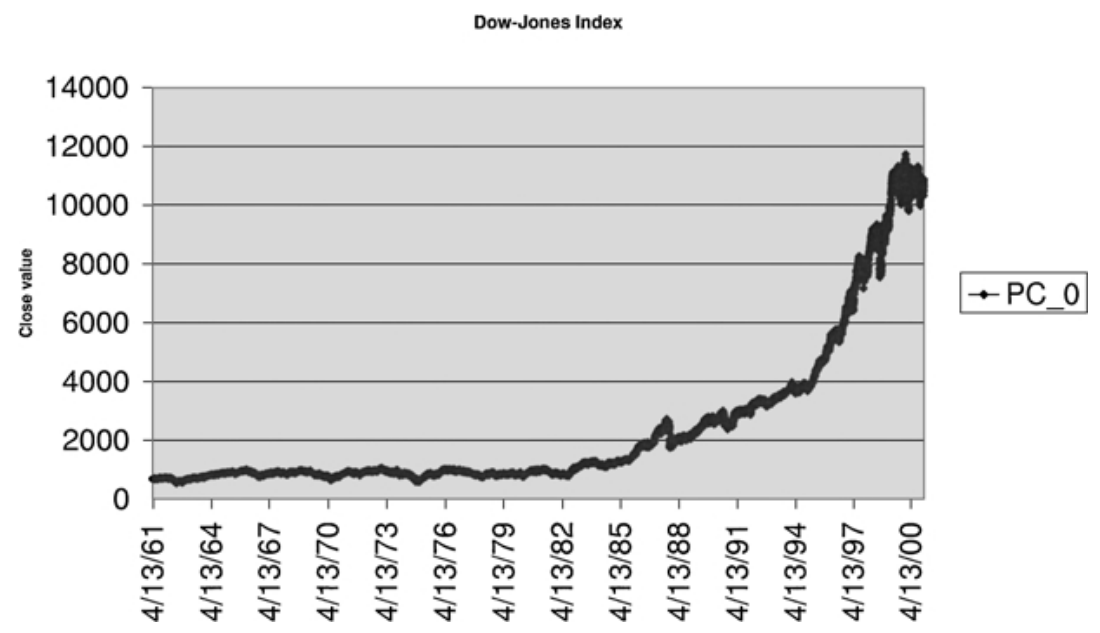

Figure 3. Dow Jones stock index, close value from April 1961 until December 2000.

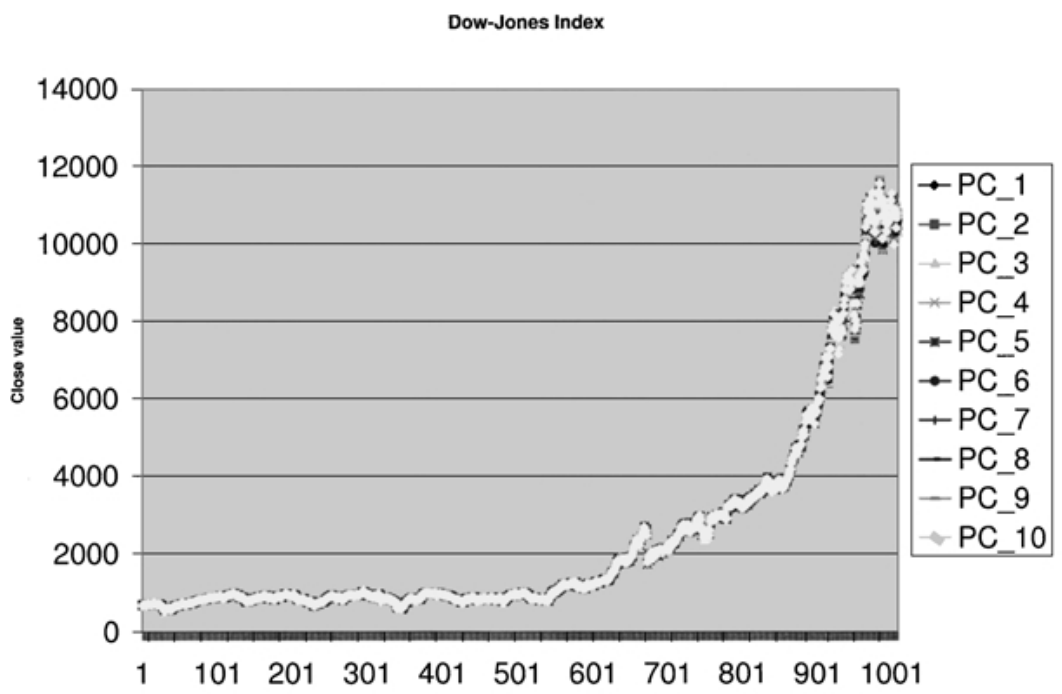

Figure 4. Dow-Jones stock index distributed over 10 computers using DWS technique.

seen at any point during query evaluation is a uniform random sample of the underlying data.

\subsection{Query distribution and result merging}

Typical OLAP queries are executed in a distributed way by all the computers that constitute the DWS system (further on we will see the query modifications required to distribute 


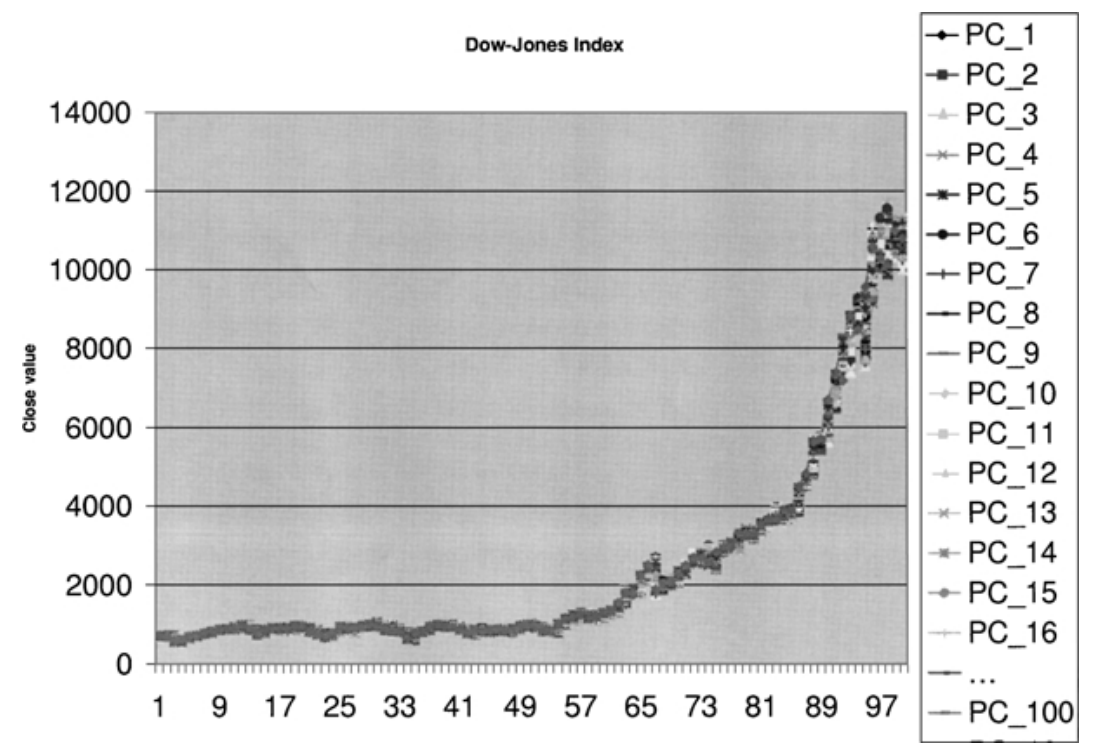

Figure 5. Dow-Jones stock index distributed over 100 computers using DWS technique.

the queries over all the computers). Two major goals can be achieved by the DWS approach:

Speedup: the execution time of typical OLAP queries can be greatly reduced. As we will see, using DWS it is possible to achieve a nearly optimal speedup for most of the OLAP queries (i.e., for example, if the star is striped into five computers the query execution time is reduced nearly five times).

Scaleup: a very large number of computers can be used which means DWS represents an effective way to handle very large data warehouses. The only limiting factor for the number of computers that can be used in a DWS system is the overhead due to the computation of the final query result from the partial results. However, for typical OLAP queries this overhead is small, which means it does not represent a problem even when a large number of computers are used.

In DWS, typical OLAP queries are transformed into $N$ partial queries that are executed in parallel in each of $N$ computers that represent the DWS system (see figure 6), avoiding the need of communication between computers during query execution. The parallel execution of a query by the available computers maintains the best load balance because the number of fact rows stored in each computer is about the same (each computer has $1 / N \pm 1$ of the total amount of fact rows in the star, with $N$ being the number of computers).

The Query Distribution and Processing (QDP) is a layer that must exist in all computers of a DWS system and is responsible to receive the original query from the client application, rewriting it if necessary, and distribute this "modified" query by all computers. After the 


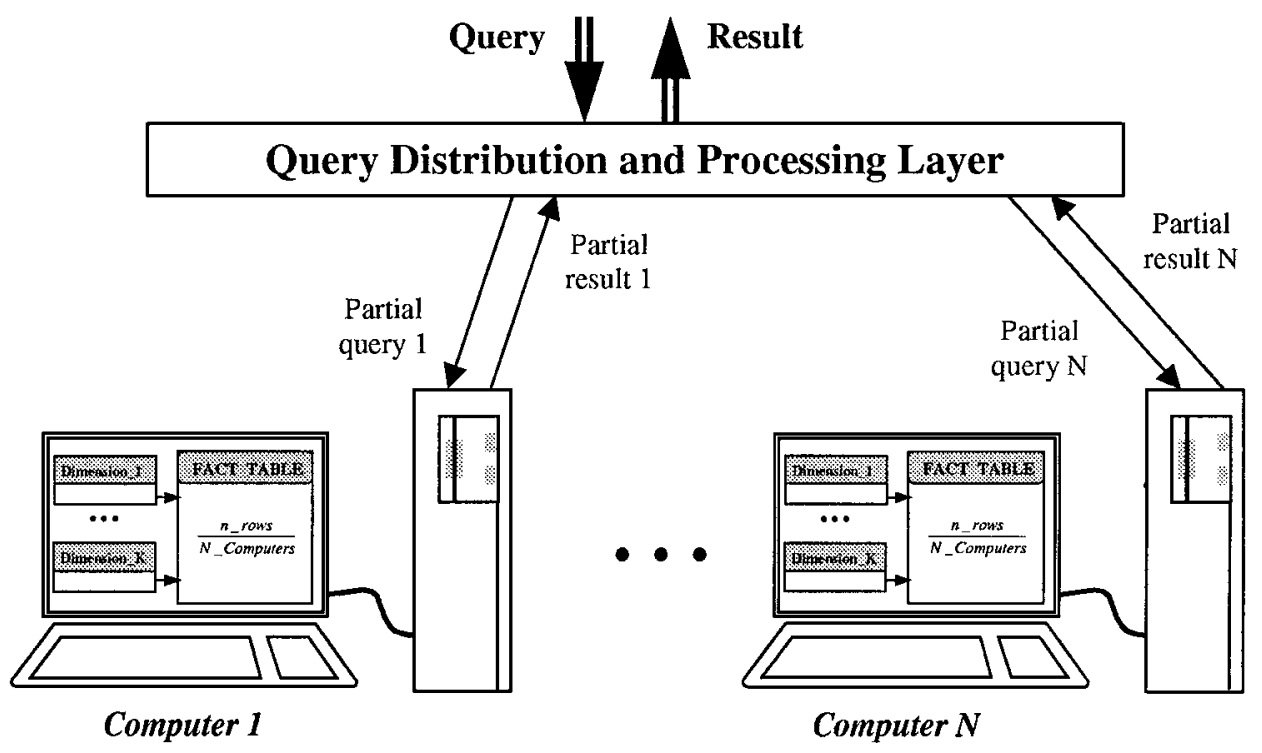

Figure 6. Query distribution.

execution of these partial queries, the QDP layer merges the partial results and sends the final result to the user.

A key aspect of DWS is the query classification and modification necessary to distribute the original query to the DWS nodes. Following, we analyze the most relevant types of queries, concerning the required modifications.

Queries containing the sum and count aggregation functions don't need to be rewritten. The partial results returned from each computer must be regrouped to compute the final result. The final result of the queries with aggregation functions is computed using the following formulas, where $N=$ number of computers used in the approach and (SUM, COUNT, AVG, VARIANCE, MAX, MIN $)_{i}$ is the aggregation function result of the set elements of computer $i$.

$$
\begin{aligned}
& S U M_{\text {total }}=S U M_{\text {partial }_{1}}+S U M_{\text {partial }_{N}}=\sum_{i=1}^{N} S U M_{i} \\
& \operatorname{COUNT}_{\text {total }}=\mathrm{COUNT}_{\text {partial }_{1}}+\mathrm{COUNT}_{\text {partial }_{N}}=\sum_{i=1}^{N} S U M_{i} \\
& A V G_{\text {total }}=\frac{S U M_{\text {partial }_{1}}+\cdots+\text { SUM }_{\text {partial }_{N}}}{\operatorname{COUNT}_{\text {partial }_{1}}+\cdots+\operatorname{COUNT}_{\text {partial }_{N}}}=\frac{\sum_{i=1}^{N} S U M_{i}}{\sum_{i=1}^{N} C O U N T_{i}} \\
& V A R_{\text {total }}=\frac{\sum_{i=1}^{N}\left[\left(\operatorname{COUNT}_{i}-1\right) V A R_{i}+\operatorname{COUNT}_{i} A V G_{i}^{2}\right]-\frac{\left(\sum_{i=1}^{N} \operatorname{COUNT}_{i} A V G_{i}\right)^{2}}{\sum_{i=1}^{N} \operatorname{COUNT}_{i}}}{\sum_{i=1}^{N} \operatorname{COUNT}_{i}-1} \\
& S T D D E V_{\text {total }}=\sqrt{V A R_{\text {total }}}
\end{aligned}
$$


The HAVING clause can be used to specify restriction over the groups. This clause only exists if we have the GROUP BY clause. The HAVING clause is removed from the original query and the group restriction is only applied after regrouping the partial results from all DWS nodes.

The ORDER BY clause, if it exists in the original query, could either be sent or not to the DWS nodes. If we send the original query without modification, i.e. with ORDER BY clause, this means that each computer orders its partial set. This option means that each computer has more processing time but the merging phase has less work. In both cases the result must be ordered in the merging phase.

A class of SQL queries that must be rewritten before it is sent to DWS system are the queries that contain the aggregation functions $A V G, S T D D E V$ and VARIANCE. The following examples illustrate the modifications of each one of the cases showing the original $(\odot)$ and the modified query $(\mapsto)$ :

$\odot$ select $A V G($ attribute) from table

$\mapsto$ select SUM(attribute), COUNT(attribute) from table

$\odot$ select STDDEV(attribute) from table

$\mapsto$ select COUNT(attribute), VARIANCE(attribute), AVG(attribute) from table

$\odot$ select VARIANCE(attribute) from table

$\mapsto$ select COUNT(attribute), VARIANCE(attribute), AVG(attribute) from table

A very important advantage of the DWS technique is that it can be used with the commercial systems available today for data warehousing, as it does not require any particular change in the database engines. The specific software layer required (QDP) can be added as an external layer (external from the database engine point of view) to the system, as all that is required is to capture the queries received, dispatch them to the DWS nodes, and compute the final result from the partial results received from the DWS nodes.

\subsection{DWS speedup}

Although this is not the main point of this paper, it is important to mention that DWS achieves an optimal speedup as was thoroughly evaluated in a previous work (Bernardino and Madeira, 2001). We have made experiments with 3, 5 and 10 workstations using a set of typical queries from APB-1 benchmark (OLAP Council, 1998). Figure 7 shows the average speedup obtained for the set of typical queries using different configurations (different number of workstations) when compared to the query execution time when the data warehouse is centralized in only one workstation.

These results show an optimal speedup for all configurations. In fact, the speedup is higher than the theoretical value, because the centralized data warehouse that was used as the reference experiment worked near the workstation memory and I/O limits. Although the speedup increases as the number of workstations used in a DWS system increases, the probability of unavailability of a subset of those workstations also increases proportionally. 
Data Warehouse Striping Speedup

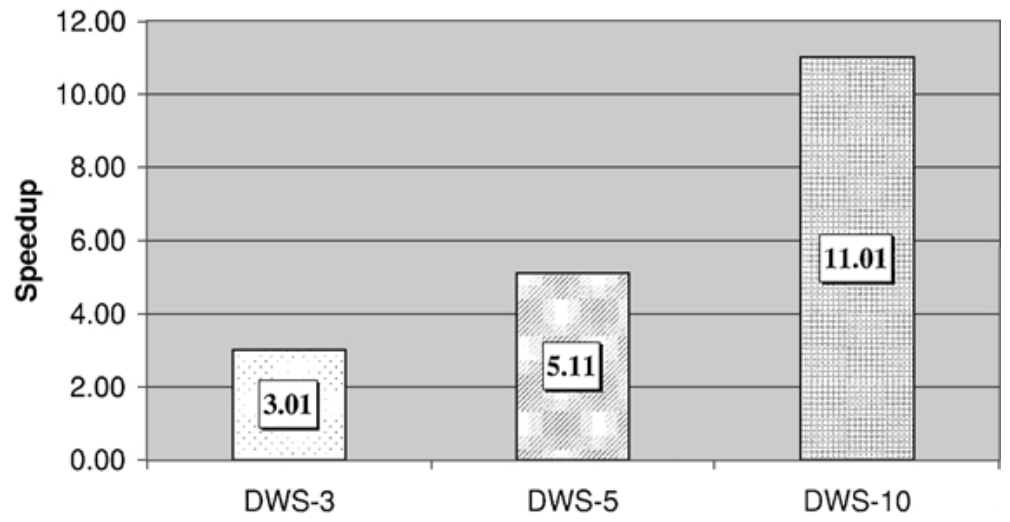

Figure 7. DWS average speedup using 3, 5 and 10 workstations.

In practice, this means that DWS could not be used with a large number of workstations because it would become unstable. This problem therefore would impair the use of this simple technique. However, we propose a radical solution to this problem, which allows even ordinary computers to be used as DWS computing units (without any special characteristic of hardware or software fault tolerance). In the proposed solution, the system continues running normally, even if one or more workstations are unavailable, and an approximate answer is computed from those that are available. Additionally, confidence intervals are also returned with the estimated result.

We experimentally analyzed our proposal with different configurations (different number of workstations) and in different scenarios of unavailability. The extreme case of unavailability will also be analyzed (when the user can only access his own workstation), as the approximate result obtained this way can still be useful when s/he is in the phase of "digging" the data and the precision of the result is not a crucial issue.

\subsection{Limitations of the technique}

The proposed DWS approach seems to provide a very promising contribution to the scalability and efficient processing of huge data warehouses. However, the technique has some intrinsic limitations:

- DWS is specifically targeted to data warehouses organized as star schemas. This means that this technique cannot be arbitrarily adapted to other type of databases, in particular operational databases (OLTP). DWS is suitable essentially for those data warehouses that are predominantly dominated by one or more very large fact tables;

- Typically, the dimensions of a star schema are small in size when compared with the big fact table. However, there are exceptions to this rule, in which case the space overhead of DWS becomes more significant; 
- If the query issued by the user didn't use the fact table but only the dimension tables, the speedup of the approach is equal to one. Of course, this is not normal because users usually want numeric measures that are contained in fact tables;

- Correlated queries which use references from outer query blocks cannot be handled directly by this approach. To overcome this problem one solution is to use query decorrelation, as proposed in literature (Rao and Ross, 1998; Seshadri et al., 1996). Using these techniques it is possible to rewrite the correlated query in such a way that outer references no longer exists. One problem with the rewriting strategy is that query decorrelation is not always possible and in some cases, although possible, it may not be efficient.

\section{Approximate query answering in the presence of node failures}

Our proposal consists of providing the user with an answer even when one of the machines in the DWS system has a failure. In this case the answer will be approximate, because some partial results of the failed machines are unavailable. DWS is working normally with approximate answers until we manually recover the workstation. However, we show that this solution is acceptable for the following reasons:

- The error is very small, as will be shown in this paper and a small error is not relevant for the decision support activities in most cases;

- It is possible to provide the user with a confidence interval that gives him/her an idea of the amount of error in the approximate result.

For queries using aggregation functions an approximate answer is simply an estimated value for the answer given together with an accuracy value in the form of confidence intervals. We provide confidence intervals based on large sample bounds (Haas, 1997). Large sample bounds contain the final answer with a specified probability and are based on Central Limit Theorem.

\subsection{Estimated values}

In this section, we will see how DWS compute the approximate aggregation values when one or more workstations cannot contribute to the final result. Consider the number of workstations used in DWS to be $N=N_{u}+N_{a}$, where $N_{u}$ is the number of workstations that are unavailable and $N_{a}$ is the number of workstations that are available, contributing to compute the estimated aggregation value. If the aggregation function to compute is AVERAGE and one or more workstations are unavailable the approximate average are simply given by

$$
A V E R A G E_{\text {estimated }}=\frac{S U M_{a}+N_{u} \frac{S U M_{a}}{N_{a}}}{\operatorname{COUNT}_{a}+N_{u} \frac{\operatorname{COUNT_{a}}}{N_{a}}}=\frac{S U M_{a}}{\operatorname{COUNT_{a}}}
$$


where $S_{U} M_{a}$ and $\operatorname{COUNT}_{a}$ represents the partial sum and count from the available workstations. Intuitively, the overall estimated average is same as the average taken from the available nodes.

For the SUM and COUNT aggregation functions the final result are basically computed as:

$$
\begin{aligned}
\operatorname{SUM}_{\text {estimated }} & =S U M_{a}+N_{u} \frac{S U M_{a}}{N_{a}}=S U M_{a} \frac{N}{N_{a}} \\
\operatorname{COUNT}_{\text {estimated }} & =\operatorname{COUNT}_{a} \frac{N}{N_{a}}
\end{aligned}
$$

where $N$ is the number of workstations used in the DWS system. These are the formulas that will be used in our experiments to compute the estimated values of the queries.

\subsection{Analysis of the error incurred in DWS estimations}

When one or more workstations are unavailable, approximate query answers must be given. Although it is not possible to return exact answers in those cases, the estimation is extremely accurate for an important subset of typical query patterns consisting of aggregations of values into categories. The estimation is based in statistical inference using the available data as samples. We assume that the random sample is taken from an arbitrary distribution with unknown mean $\mu$ and unknown variance $\sigma^{2}$. We make the additional assumption that the sample size $n_{s}$ is large enough $\left(n_{s}>30\right)$ so that the Central Limit Theorem can be applied and it is possible to make inferences concerning the mean of the distribution (Cochran, 1977). As $\sigma$ is unknown, we replace it with the estimate $s$, the sample standard deviation, since this value is close to $\sigma$ with high probability for large values of $n_{s}$. Thus, bounds on the confidence interval for the mean of an arbitrary distribution are given by $\bar{X} \pm$ Error, where

$$
\text { Error }= \pm Z_{\alpha / 2} \times \frac{s}{\sqrt{n_{s}}} \sqrt{\frac{n-n_{s}}{n-1}} .
$$

In this expression, $s$ is the standard deviation of the sample and $n$ is the population size. The term $Z_{\alpha / 2}$ is the corresponding percentile in the normal distribution. This expression shows that the error decreases significantly as the sample size $n_{s}$ increases and eventually reaches extremely small values for very large sample sizes $\left(n_{s} \approx n\right)$.

The distribution of the fact table rows into $N$ workstations is considered uniform because as we have shown in Section 3.1 the round-robin approach is a random sample method. As a result, we assume the values in any aggregation pattern to be evenly distributed by all the workstations. For simplicity, we consider that an average is being computed over each aggregation group. We also consider that $N_{u}$ workstations are unavailable (cannot contribute with partial values to the final query result). Some reasonable assumptions can be made concerning the values taken by these variables,

- $1<N \leq 100$

- $N_{u}$ is typically a small fraction of $N$. 

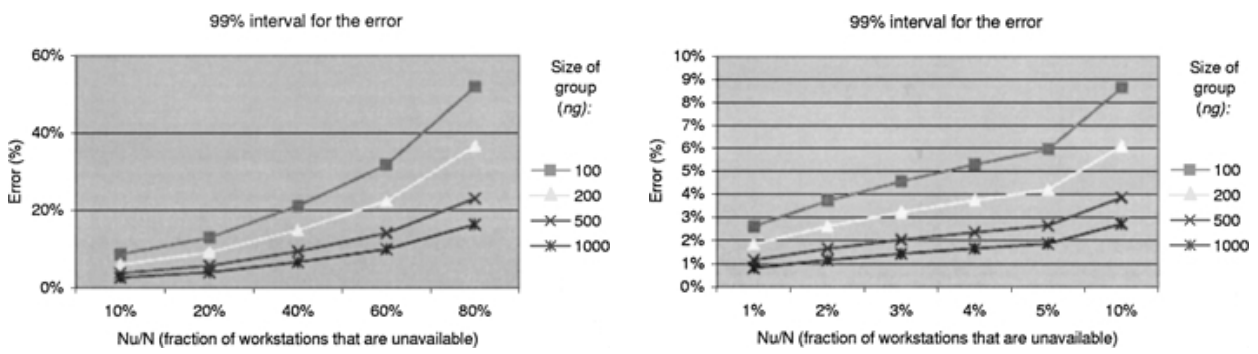

Figure $8.99 \%$ Confidence intervals for the error.

Consider also an aggregation of $n_{g}$ values into one of the groups of the result, with $n_{g}$ being reasonably large (e.g. $\left.n_{g} \geq 100\right)$. The number of values available in the sample when $N_{u}$ workstations are unavailable is $n_{g}-n_{g} / N \times N_{u}=n_{g}\left(1-N_{u} / N\right)$ and the error is:

$$
\begin{aligned}
\text { Error } & = \pm Z_{\alpha / 2} \times \frac{s}{\sqrt{n_{g}\left(1-N_{u} / N\right)}} \sqrt{\frac{n_{g}-n_{g}\left(1-N_{u} / N\right)}{n_{g}-1}} \\
& \approx \pm Z_{\alpha / 2} \times \frac{s}{\sqrt{n_{g}\left(1-N_{u} / N\right)}} .
\end{aligned}
$$

This value is typically extremely small for the type of queries considered, because the number of values aggregated into a specific group $\left(n_{g}\right)$ is at least reasonably large (e.g. more than 100 values) and the fraction $N_{u} / N$ is usually small. In other words, a sufficiently large sample is usually available, resulting in very accurate estimations. In these formulas we are concerning about the mean of the distribution but if we would like to compute the sum or count is only multiply the formulas above by the number of elements in each group $\left(n_{g}\right)$. Figure 8 shows the $99 \%$ interval for the error taken as a function of the fraction of workstations that are unavailable ( $x$ axis) and considering also different numbers of values aggregated in a group. These results were obtained by considering the standardized normal distribution $\mathcal{N}(0,1)$.

The results of the figure 8 (left) show that the error is very small when the fraction of workstations that are unavailable is reasonably small or the number of values aggregated into the group is sufficiently large. Additionally, although the increase in the fraction of workstations that are unavailable results in larger errors, as shown in the figure 8 (right), those errors are not very large in many cases and decrease significantly as $n_{g}$ increases. For instance, it can be seen from the left picture that the error is extremely small when the fraction of unavailable workstations is less or equal to $10 \%$ and the number of values aggregated into the group is larger than 200 .

\section{Experimental results}

In the experiments we evaluated the error and test if it is within the estimated confidence intervals in a large variety of cases. We are also interested in analyzing the influence of group-by queries with different sizes of groups. 
Table 1. Characteristics of queries Q1, Q6 and Q7.

\begin{tabular}{lrcc}
\hline & Number of rows/group & Number of groups & Selectivity (\%) \\
\hline Q1 & $1,479,417$ & 4 & 14.6 \\
Q6 & 114,160 & 1 & 1.9 \\
Q7 & 1,481 & 4 & 0.025 \\
\hline
\end{tabular}

The TPC-H benchmark (TPC, 1999) was used as an example of a typical OLAP application. It consists of a suite of business oriented ad hoc queries illustrating decision support systems that examine large volumes of data, execute queries with a high degree of complexity and give answers to critical business questions. Therefore, in accordance with the above criteria, we concentrated our attention on queries Q1, Q6 and Q7 of TPC-H benchmark. The definition of the queries with its SQL code and results are shown in the Appendix.

These queries have the characteristics shown in Table 1, where the first column represents the number of rows processed in average for each group, the second column show the number of groups and the third represents the average group selectivity when the data warehouse is centralized in one workstation.

\subsection{Experimental testbed}

The experimental evaluation of approximate query answering in DWS is conducted in a workstation environment where all are linked together in an Ethernet network with Oracle 8 database management system installed in each of them.

The TPC-H was implemented with a scale factor of 1 for the test database, which corresponds, to approximately $1 \mathrm{~GB}$ for the database size. This corresponds to a big fact table LINEITEM (6,001,215 rows) and the dimension tables ORDERS (1,500,000 rows), CUSTOMER (150,000 rows), SUPPLIER (10,000 rows) and NATION (25 rows) as shown in figure 9.

In these experiments we apply our technique to one workstation, simulating a centralized data warehouse and denote it as CDW (Centralized Data Warehouse), and to $N=5,10,20,50$ and 100 workstations, which corresponds to DWS-5, DWS-10, DWS-20, DWS-50 and DWS-100, respectively.

The use of $N$ workstations was simulated by dividing the $n_{-}$fact_rows $(6,001,215)$ of fact table, into $N$ partial fact tables (LINEITEM_1, $\ldots$, LINEITEM_N). Each workstation has $n_{-}$fact_rows $/ N$ rows and the dimensions are replicated in each workstation. For example, DWS-100 simulates the use of 100 workstations $(N=100)$ having 100 partial fact tables (LINEITEM_1, ., LINEITEM_100) with each line having 60, 021 \pm 1 fact rows, while the dimensions are equivalent to those of the CDW system.

\subsection{Approximate query answers}

In these experiments we evaluated the error obtained with typical OLAP queries when some of the workstations are unavailable and proved that we can give very tight confidence 


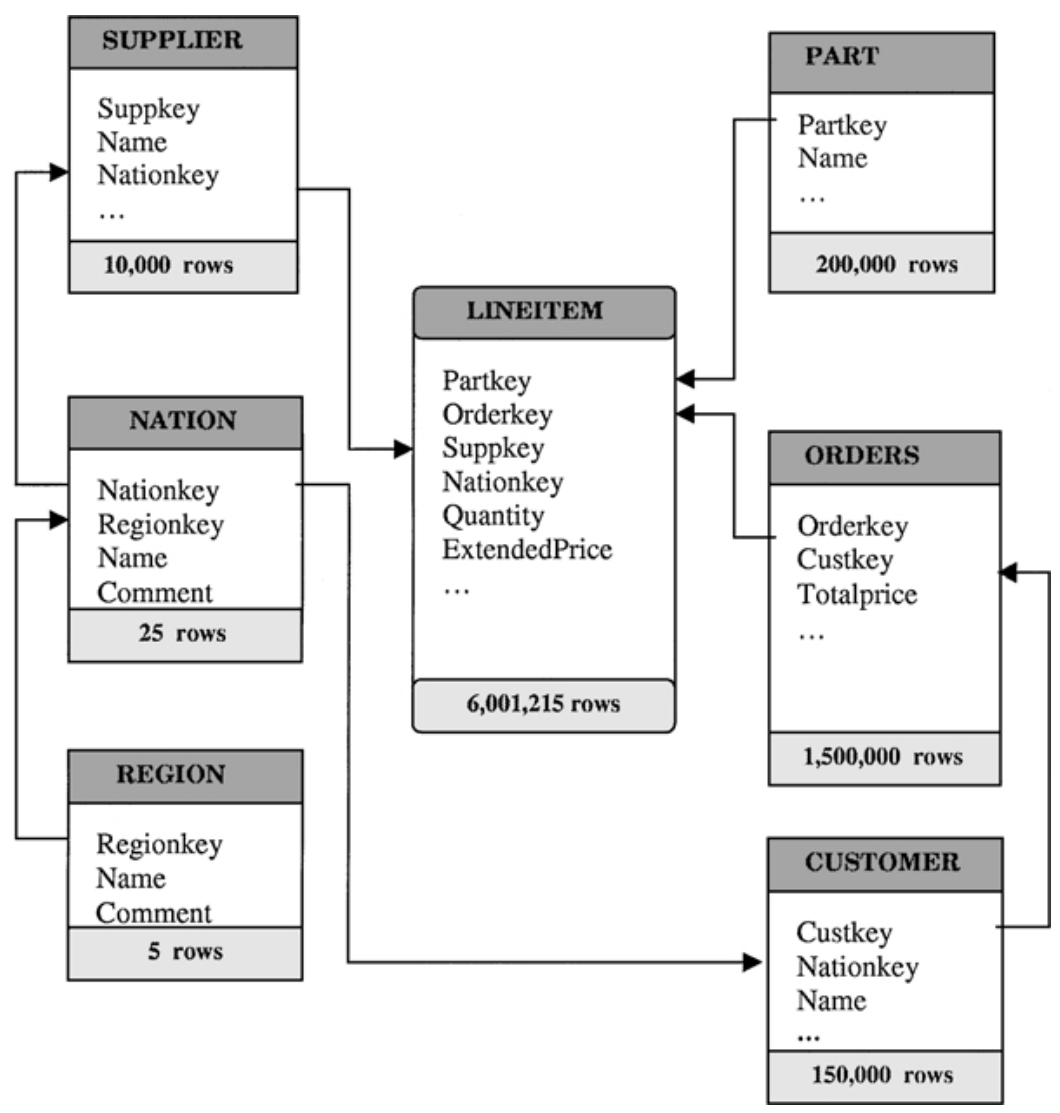

Figure 9. TPC-H benchmark schema.

intervals such that users know about the accuracy of the result. The influence of group-by queries with different sizes of groups will also be analyzed.

5.2.1. Estimation accuracy. The unavailability of each individual workstation was simulated by not taking into account the partial results that corresponded to the failed workstation. Finally, we compute the average and maximum of all these errors for each configuration. For example, using the DWS-100 configuration, we determine the error when one of the 100 workstations is unavailable and determine the average and maximum of these errors.

The average and maximum error obtained for Q1, Q6 and Q7 queries of TPC-H benchmark using DWS-5, DWS-10, DWS-20, DWS-50 and DWS-100 and considering only one unavailable workstation are illustrated in figure 10, where the $x$ axis represents the number of workstations used.

As can be observed in figure 10 (left), the average error obtained for these queries is extremely small because we only simulate the unavailability of one of the workstations 

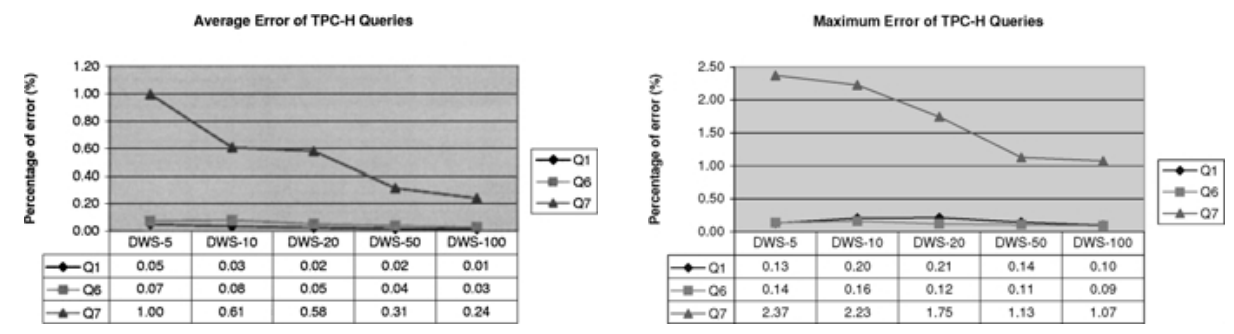

Figure 10. Average and maximum error for Q1, Q6 and Q7 when one workstation is unavailable.

that comprise each configuration. Additionally, this error decreases when we use more workstations, due to the fact that the number of missing rows is smaller. The average error obtained for Q7 query is larger than the error corresponding to the other queries because the average number of rows aggregated by group is smaller. This is due to the fact the query Q7 has a higher selectivity (as shown in Table 1), meaning less elements in each aggregation group, which in case of failure of one workstation has more impact in the precision of the result obtained.

The maximum error is higher than average error because it is the worst-case. We compute the maximum error obtained for each query and for each group. However, as illustrated in the right picture of figure 10, this error was always smaller than $2.5 \%$ even when $1 / 5$ of workstations were unavailable.

In the resutls shown before only one workstation was unavailable, but we are also interested in studying the results when the number of unavailable workstations is much larger. For instance, in DWS-5 we can simulate the failure of 2, 3 or 4 workstations, which corresponds to an unavailability of $40 \%, 60 \%$ or $80 \%$, respectively. The average and maximum error for all possible combinations is shown in figure 11 for queries Q1, Q6 and Q7, where the $x$ axis represents the number of workstations unavailable.

In these experiments, using DWS-5 configuration, the error increases with the number of workstations that are unavailable (as expected). However, this error is not very large in average, as it does not exceed 4\% (Q7 query) or even less. Furthermore, the average error is not higher than $0.3 \%$ for queries Q1 and Q6, which is a very good approximation of the result for most of the cases (see figure 11, left). These very good results are due to the fact
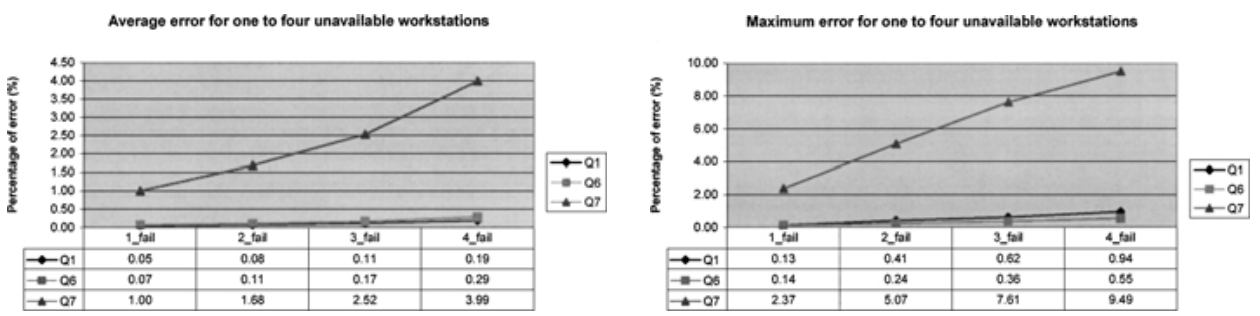

Figure 11. Average and maximum error on DWS-5 when one to four workstations are unavailable. 
that our partitioning strategy is pseudo-random resulting in a uniform random distribution of the data over the workstations.

The maximum error obtained using all the possible combinations of workstation unavailability is about 10\% (see figure 11, right). It must be pointed out this is the extreme case of unavailability because the user is only accessing his own workstation.

Interestingly, the maximum error of Q1 query is higher than the maximum error of Q6 even though Q1 aggregates more rows in average than Q6 (see Table 1). However, this is due to the influence of a very small group in Q1. This group has 38,854 rows, which is a much smaller number of rows than those from query Q6 (see Table 1). Therefore, we could conclude that the precision of our results is highly influenced by the number of rows processed in each group of a group-by query. However, even in the case of unavailability of $80 \%$ of the workstations we obtain an error less than $10 \%$ in the worst case meaning that approximate results are not harmful.

5.2.2. Confidence intervals. We provide accuracy measures as confidence intervals for each estimate, for some confidence probability.

The next figures analyze the confidence interval that is returned using our technique for queries Q1 and Q7 using various configurations of DWS. Each graphic shows three curves, two of them representing the sum of the exact value with the confidence interval (exact_value + error and exact_value - error), corresponding to the upper and lower curves in the figures. The middle curve is the estimated_value obtained with the respective configuration.

Figure 12(left) shows the confidence interval for query Q1 using DWS-100 and the aggregation avg(l_extendedprice) for one of the groups of the result. As we are simulating the unavailability of only one workstation, the $x$ axis legend indicates which workstation is unavailable and the $y$ axis shows the value of the aggregation as well as the confidence interval bounds. This example shows that the confidence intervals do a good job determining boundaries for the error. These intervals are computed using the Eq. (5) of Section 4.2, with a probability of $99 \%$.

Figure 12(right) shows the confidence intervals for query Q7 and all possible combinations of unavailability of three workstations using DWS-5. The value computed by query Q7 is the aggregation sum(volume). The $x$ axis represents all possible combinations of unavailability of three workstations. The query Q7 returns four groups, but for simplicity is only shown the result of one in the figure 12(right). In this case confidence intervals are computed with a probability of $95 \%$.
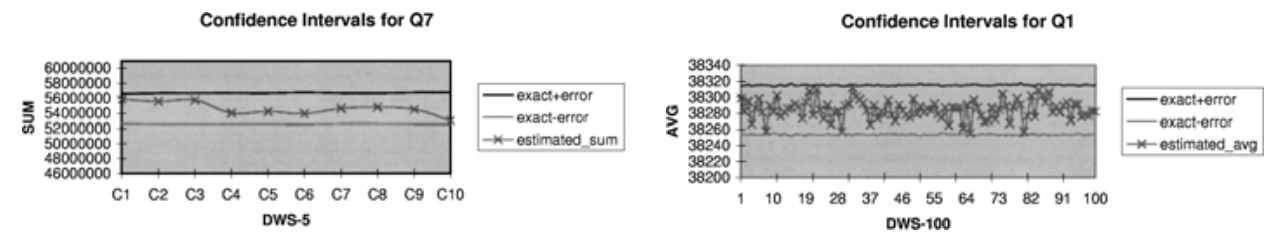

Figure 12. Confidence interval for queries Q1 and Q7 using DWS-100 and DWS-5. 
The experimental results show that confidence intervals are very useful to deliver convenient error bounds for a given confidence level and the errors are very small. Thus, we can give the user very tight confidence intervals of the approximate answers when one or more workstations are unavailable. The artificial nature of the TPC-H benchmark data could influence the results. However, we argue that these highly accurate answers are mainly due to the round robin data partitioning which ensures a uniform distribution of fact data over all computers, which would not be the case if we have used range partitioning of fact data.

\section{Conclusions}

Data Warehouse Striping (DWS) is a scalable technique that divides data warehouse facts into a number of workstations to solve data warehouse limitations related to heavy storage loads and performance problems. In this paper, we proposed an approach to deal with the problem of the unavailability of one or more computers in the cluster, allowing the use of DWS technique with a very large number of inexpensive computers.

With the proposed modular approach, simple workstations without any special hardware or software fault tolerance can be used and very accurate approximate answers are returned even when a substantial number of the component workstations are unavailable. We have proposed formulas to quantify estimation error of the answer and proved that this error is very small when the fraction of workstations that are unavailable is reasonably small or the number of values aggregated into the groups is sufficiently large.

The proposed technique is a cost-effective solution that could be applied in almost all types of organizations, taking advantage of the availability of computer networks to distribute the data and their processing power, avoiding the need of very expensive servers. The experimental results show a linear or even super linear speedup of DWS, due to the fact that, when we distribute the data, we are working with more manageable amounts of data that do not stress memory and computing resources so much.

The experimental results of this paper have also shown that the DWS technique provides approximate query answers with very small errors, even when most of the workstations are unavailable. The confidence intervals are promising, as the technique is able to return strict confidence intervals because the data warehouse facts are partitioned in a strict uniform way, providing the user with a measure of the accuracy of the query results.

\section{Appendix: TPC-H benchmark queries}

Query 1: Pricing summary report

This query reports the amount of business that was billed, shipped and returned. The Pricing Summary Report Query provides a summary pricing report for all Lineitems shipped as of a given date. This query performs multiple aggregations and summaries by reading and processing over $95 \%$ of the rows of the database's largest table (LINEITEM). Only this single table is scanned with a very low number of rows being returned. 


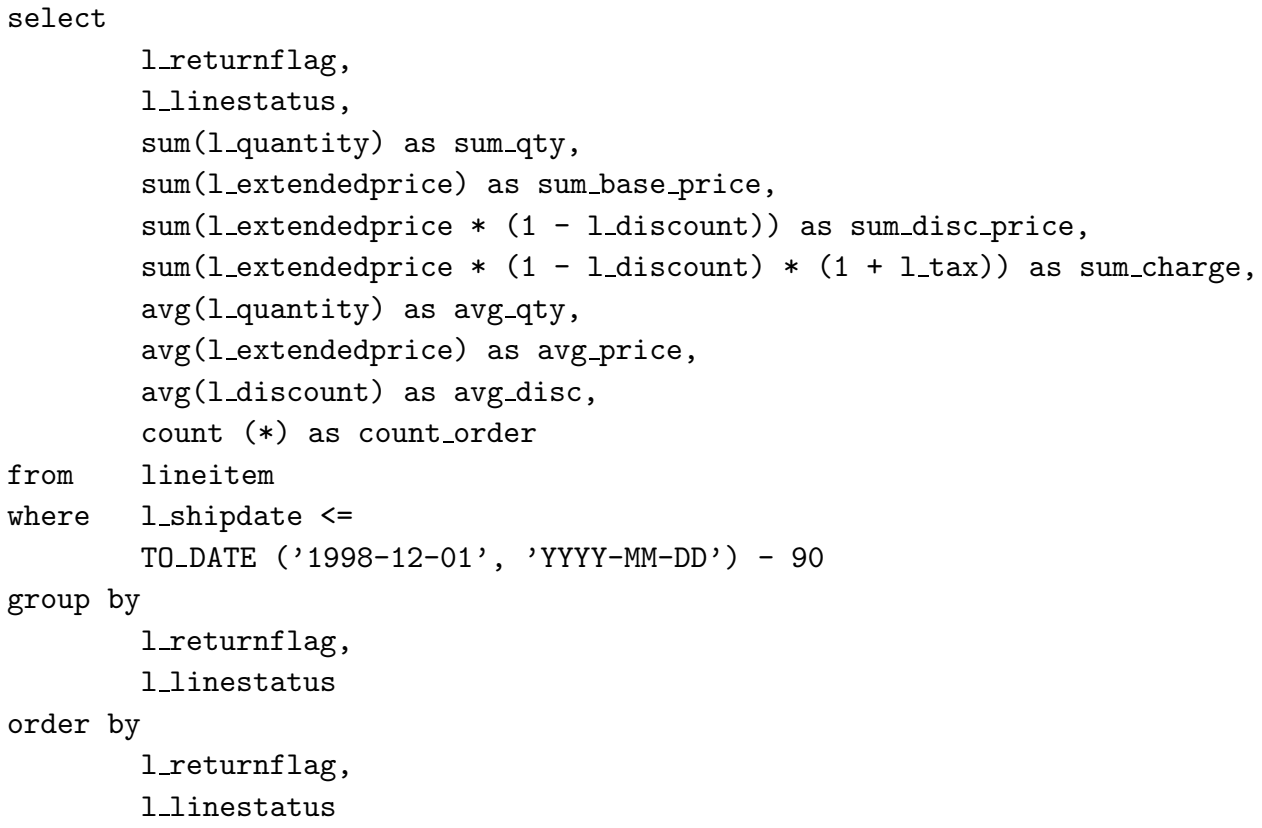

Query output data:

\begin{tabular}{llllllllll}
\hline L_RETURNFLAG & L_LINESTATUS & SUM_QTY & SUM_BASE_PRICE & SUM_DISC_PRICE & SUM_CHARGE & AVG_QTY & AVG_PRICE & AVG_DISC & COUNT_ORDER \\
\hline $\mathrm{A}$ & $\mathrm{F}$ & 37734107.00 & 56586554400.73 & 53758257134.87 & 55909065222.83 & 25.52 & 38273.13 & 0.05 & 1478493 \\
$\mathrm{~N}$ & $\mathrm{~F}$ & 991417.00 & 1487504710.38 & 1413082168.05 & 1469648223.19 & 25.52 & 38284.47 & 0.05 & 38854 \\
$\mathrm{~N}$ & $\mathrm{O}$ & 74476040.00 & 117701729697.74 & 106118230307.61 & 110367043872.50 & 25.50 & 38249.12 & 0.05 & 2920374 \\
$\mathrm{R}$ & $\mathrm{F}$ & 37719753.00 & 56568041380.90 & 53741292684.60 & 55889619119.83 & 25.51 & 38250.86 & 0.05 & 1478870 \\
\hline
\end{tabular}

Query 6: Forecasting revenue change

This query quantifies the amount of revenue increase that would have resulted from eliminating certain company-wide discounts in a given percentage range in a given year. Asking this type of "what-if" query can be used to look for ways to increase revenues. This query accesses the large detail table only (LINEITEM) selecting about $12 \%$ of the rows, and returning a single column answer.

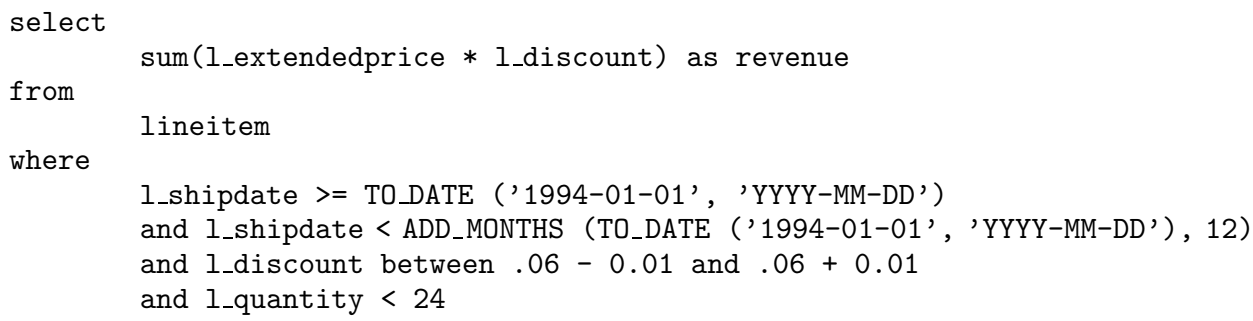


Query output data:

$$
\frac{\overline{\text { REVENUE }}}{123141078.23}
$$

Query 7: Volume shipping

This query determines the value of goods shipped between certain nations to help in the re-negotiation of shipping contacts. This is a 6-table join that requires a small 25-row table, $N A T I O N$, to be aliased and processed as though it were two distinct look-up tables. A date constraint select $2 / 7$ of the LINEITEM rows, while a join to the lookup tables results in 2/5 of the CUSTOMERS and SUPPLIERS being selected. Four rows are returned.

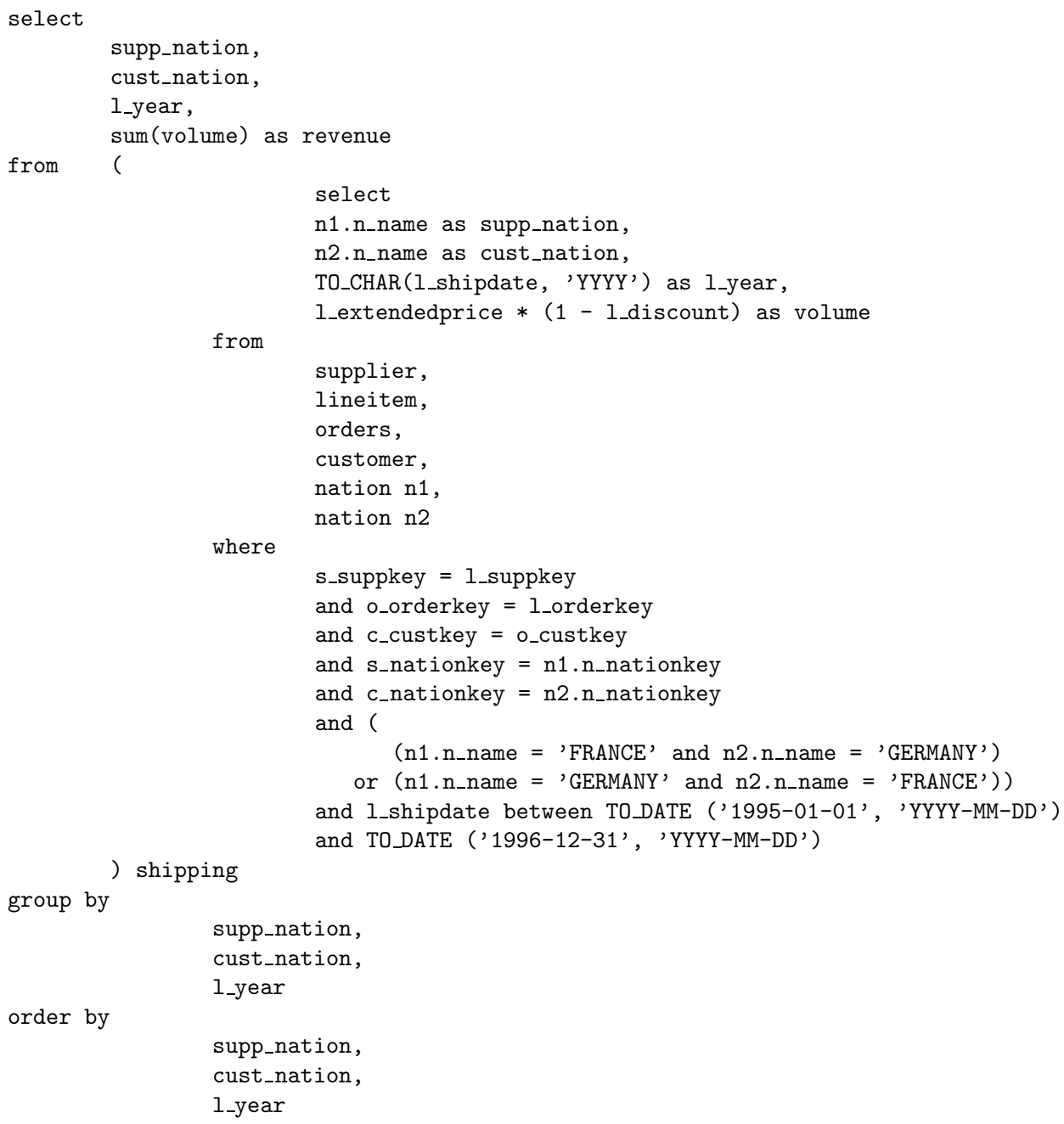


Query output data:

\begin{tabular}{llll}
\hline SUPP_NATION & CUST_NATION & YEAR & REVENUE \\
\hline FRANCE & GERMANY & 1995 & 54639732.73 \\
FRANCE & GERMANY & 1996 & 54633083.31 \\
GERMANY & FRANCE & 1995 & 52531746.67 \\
GERMANY & FRANCE & 1996 & 52520549.02 \\
\hline
\end{tabular}

\section{References}

Acharaya, S., Gibbons, P., and Poosala, V. (2000). Congressional Samples for Approximate Answering of Groupby-Queries. In Proc. of ACM SIGMOD Int. Conf. on Management of Data, Dallas, Texas, USA (pp. 487-498). Albrecht, J., Gunzel, H., and Lehner, W. (1998). An Architecture for Distributed OLAP. In Int. Conf. on Parallel and Distributed Processing Techniques and Applications (PDPTA), Las Vegas, USA.

Barbara, D. et al. (1997). The New Jersey Data Reduction Report. Bulletin of the Technical Committee on Data Engineering, 20(4), 3-45.

Bernardino, J. and Madeira, H. (2000). A New Technique to Speedup Queries in Data Warehousing. In Proc. of Chalenges ADBIS-DASFA A Symposium on Advances in Databases and Information Systems, Prague, Czech Republic (pp. 21-32).

Bernardino, J. and Madeira, H. (2001). Experimental Evaluation of a New Distributed Partitioning Technique for Data Warehouses. In Proc. of Int. Database Engineering \& Applications Symposium IDEAS, Grenoble, France (pp. 312-321).

Chauduri, S. and Dayal, U. (1997). An Overview of Data Warehousing and OLAP Technology. SIGMOD Record, 26(1), 65-74.

Chen, C.M. and Roussopoulos, N. (1994). Adaptive Selectivity Estimation Using Query Feedback. In Proc. ACM SIGMOD Int. Conf. on Management of Data (pp. 161-172).

Cochran, W.G. (1977). Sampling Techniques (3rd edn.). New York: John Wiley \& Sons.

Codd, E.F., Codd, S.B., and Salley, C.T. (1993). Providing OLAP (Online Analitycal Processing) to User Analysts: An IT Mandate. Technical Report, E.F. Codd \& Associates.

Datta, A., Moon, B., and Thomas, H. (1998). A Case for Parallelism in Data Warehousing and OLAP. In Proc. of the 9th Int. Conf. on Database and Expert Systems Applications DEXA Workshop (pp. 226-231).

DeWitt, D.J. et al. (1990). The Gamma Database Machine Project. IEEE Trans. Knowledge and Data Engineering, 2(1), 44-62.

DeWitt, D.J. and Gray, J. (1992). Parallel Database Systems: The Future of High Performance Database Systems. Communications of the ACM, 35(6), 85-98.

Ganguly, S., Gibbons, P.B., Matias, Y., and Silberschatz, A. (1996). Bifocal Sampling for Skew-Resistant Join Size Estimation. In Proc. ACM SIGMOD Int. Conf. on Management of Data (pp. 271-281).

Gibbons, P.B. and Matias, Y. (1998a). New Sampling-Based Summary Statistics for Improving Approximate Query Answers. In Proc. ACM SIGMOD Int. Conf. on Management of Data (pp. 331-342).

Gibbons, P.B. and Matias, Y. (1998b). AQUA: System and Techniques for Approximate Query Answering. Bell Labs Technical Report.

Gibbons, P.B., Matias, Y., and Poosala, V. (1997a). Aqua Project, White Paper. Technical Report, Bell Laboratories, Murray Hill, New Jersey.

Gibbons, P.B., Matias, Y., and Poosala, V. (1997b). Fast Incremental Maintenance of Approximate Histograms. In Proc. 23rd Int. Conf. on Very Large Data Bases VLDB (pp. 466-475).

Haas, P.J. (1997). Large-Sample and Deterministic Confidence Intervals for Online Aggregation. In Proc. 9th Int. Conf. on Scientific and Statistical Database Management, SSDBM (pp. 51-62).

Haas, P.J. (1999). Techniques for Online Exploration of Large Object-Relational Datasets. In Proc. 9th Int. Conf. on Scientific and Statistical Database Management, SSDBM (pp. 4-12). 
Haas, P.J., Naughton, J.F., Seshadri, S., and Stokes, L. (1995). Sampling-Based Estimation of the Number of Distinct Values of an Attribute. In Proc. 21st Int. Conf. on Very Large Data Bases VLDB (pp. 311322).

Haas, P.J., Naughton, J.F., and Swami, A.N. (1994). On the Relative Cost of Sampling for Join Selectivity Estimation. In Proc. 13th ACM Symp. on Principles of Database Systems (pp. 14-24).

Hansen, M.H., Hurwitz, W.M., and Madow, W.G. (1953). Sample Survey Methods and Theory (vols. I e II). New York: John Wiley \& Sons.

Hellerstein, J.M., Haas, P.J., and Wang, H.J. (1997). Online Aggregation. In Proc. ACM SIGMOD Int. Conf. on Management of Data (pp. 171-182).

Hou, W.-C. and Taneja, B.K. (1998). Statistical Estimators for Relational Algebra Expressions. In Proc. 7th ACM Symp. on Principles of Database Systems (pp. 276-287).

Kimball, R. (1996). The Data Warehouse Toolkit. New York: J. Wiley \& Sons.

Kimball, R., Reeves, L., Ross, M., and Thornthwalte, W. (1998). The Data Warehouse Lifecycle Toolkit. New York: J. Wiley \& Sons.

Kooi, R.P. (1980). The Optimization of Queries in Relational Databases. PhD Thesis, Case Western Reserve University.

Lipton, R.J. and Naughton, J.F. (1995). Query Size Estimation by Adaptive Sampling. J. Computer and System Sciences, 51(1), 18-25

Lipton, R.J., Naughton, J.F., and Schneider, D.A. (1990). Practical Selectivity Estimation Through Adaptive Sampling. In Proc. ACM SIGMOD Int. Conf. on Management of Data (pp. 1-11).

Lu, H., Ooi, B.C., and Tan, K.L. (1994). Query Processing in Parallel Relational Database Systems. IEEE Computer Society.

Olap Council, APB-1 Benchmark, Olap Council, November 1998, available at www.olpacouncil.org.

Olken, F. and Rotem, D. (1992). Maintenance of Materialized Views of Sampling Queries. In Proc. 8th IEEE Int. Conf. on Data Engineering ICDE (pp. 632-664).

Poosala, V. (1997). Histogram-Based Estimation Techniques in Databases. PhD Thesis, University of WisconsinMadison.

Poosala, V., Ganti, V., and Ioannidis, Y.E. (1999). Approximate Query Answering Using Histograms. IEEE Data Engineering Bulletin, 22(4), 5-14.

Poosala, V., Ioannidis, Y.E., Haas, P.J., and Shekita, E.J. (1996). Improved Histograms for Selectivity Estimation of Range Predicates. In Proc. ACM SIGMOD Int. Conf. on Management of Data (pp. 294-305).

Rao, J. and Ross, K.A. (1998). Reusing Invariants: A New Strategy for Correlated Queries. In Proc. of ACM SIGMOD Int. Conf. on Management of Data, Seattle, USA (pp. 37-48).

Selinger, P. et al. (1979). Access Path Selection in a Relational Database Management System. In Proc. ACM SIGMOD Int. Conf. on Management of Data (pp. 23-34).

Seshadri, P., Pirahesh, H., and Cliff, T.Y. (1996). Complex Query Decorrelation. In Proc. IEEE Int. Conf. on Data Engineering ICDE (pp. 450-458).

Stonebraker, M., Katz, R., Patterson, D., and Oustershout, J. (1998). The Design of XPRS. In Proc. of the Int. Conf. on Very Large Databases VLDB, Los Angeles, USA (pp. 318-330).

Transaction Processing Council (1999). TPC Benchmark H. Transaction Processing Council, June 1999, available at www.tpc.org.

Vitter, J. and Wang, M. (1999). Approximate Computation of Multidimensional Aggregates of Sparse Data Using Wavelets. In Proc. ACM SIGMOD Int. Conf. on Management of Data (pp. 193-204). 